\title{
IMPLEMENTACIÓN Y VALIDACIÓN DE PROTOCOLO DE ESTIMULACIÓN POR COMPRENSIÓN DE LENGUA- JE ORAL EN RESONANCIA MAGNÉTICA FUNCIONAL: RESULTADOS PRELIMINARES
}

Ing. Ignacio Letelier $A^{(1)}$, Drs. Steren Chabert ${ }^{(2)}$, Juan Vielma $P^{(3)}$.

1. Ingeniero Civil Biomédico, Universidad de Valparaíso, Chile.

2. Departamento Ingeniería Biomédica, Universidad de Valparaíso, Chile.

3. Neurorradiólogo, Hospital Carlos Van Buren, Valparaíso, Chile.

IMPLEMENTATION AND VALIDATION OF A STIMULATION PROTOCOL FOR ORAL LANGUAGE COMPREHENSION USING FUNCTIONAL MAGNETIC RESONANCE IMAGING: PRELIMINARY RESULTS

\begin{abstract}
This study aimed at implementing a stimulation protocol using functional Magnetic Resonance Imaging (fMRI), in a Hospital in Valparaiso (V Region), Chile, to detect cortical areas activated in oral language comprehension. Seven healthy volunteers participated in this study. Average t-score and signal variation were $6.3 \pm 0.3$ and $0.5 \pm 0.1 \%$, respectively. In spite of these low values, activations were obtained in Wernicke area, middle temporal gyrus, and Heschl's gyrus. The extension of activated areas was small, $5.06 \pm 2.99 \mathrm{~cm} 3$, probably due to amplifier low signal-to-noise ratio (SNR), in addition to the cognitive complexity of the task, and to the ambient acoustic noise. Successful implementation of fMRI protocols of language comprehension is possible in a clinical context in Chile without any additional resources.

Keywords: Functional magnetic resonance imaging, Low signal-to-noise ratio, Oral language comprehension,
\end{abstract}

Resumen: Nuestro objetivo fue implementar un protocolo de estimulación en resonancia magnética funcional en un Hospital de Valparaíso, $V$ Región de Chile, para detectar las áreas corticales activadas en la comprensión del lenguaje oral. Siete voluntarios sanos participaron de este estudio. El t-score y variación de

Letelier I y cols. Implementación y validación de protocolo de estimulación por comprensión de lenguaje oral en resonancia magnética funcional: Resultados preliminares. Rev Chil Radiol 2010: 16(2): 64-69.

Correspondencia: Ignacio Letelier A.

iletelier@csm.cl

Trabajo recibido el 20 de octubre de 2009, aceptado para publicación el 17 de mayo de 2010. señal alcanzado fue de 6.30 .3 y $0.5 \pm 0.1 \%$ respectivamente. A pesar de estos bajos valores, las activaciones se registraron en el área Wernicke, circunvolución temporal media y circunvolución de Heschl. La extensión de las activaciones fue pequeña, $5.06 \pm 2.99 \mathrm{~cm}^{3}$, probablemente debido a la baja relación señal ruido del resonador (SNR), además de la complejidad cognitiva de la tarea y el ruido acústico ambiente. Nuestros resultados indican que la implementación de la RMf en comprensión del lenguaje es posible de realizar en Chile sin recursos adicionales.

Palabras clave: Baja razón señal-ruido, Comprensión del lenguaje oral, Resonancia magnética funcional.

\section{Introducción}

La resonancia magnética funcional (RMf) permite localizar, de forma no invasiva, las áreas del cerebro activadas utilizando el contraste generado por las diferencia en los niveles de oxigenación de la sangre ${ }^{(1)}$. Los cambios hemodinámicos medidos reflejan la actividad neuronal con una sensibilidad lo suficientemente alta para estudiar las funciones que desempeñan las diferentes áreas del cerebro. Por esta razón, la RMf ha sido aplicada en pacientes con tumores cerebrales y epilepsia, para facilitar la selección de candidatos para cirugía, planear la operación y mejorar la orientación intraoperatoria ${ }^{(2,3)}$. Los diferentes estudios realizados en cuanto al procesamiento del lenguaje mediante resonancia funcional se han centrado en la investigación de las áreas involucradas principalmente en el procesamiento de palabras y frases, utilizando estimulación auditiva y visual ${ }^{(4,5)}$. Los resultados obtenidos han dado cuenta de una extensa red de áreas entre las que se encuentran las de comprensión y producción del lenguaje, es decir, Wernicke y Broca respectivamente, región temporal posterior y circunvoluciones temporales ${ }^{(6,7,8)}$.

Además de las palabras y las frases, también se utilizan historias como método de estimulación. Tzourio et al ${ }^{(9)}$, desarrollaron un protocolo que consistió en que 
los voluntarios escucharan una historia de forma pasiva; la tarea auditiva se contrastó con una condición de no activación. Este método permitió activar los lóbulos temporales de forma bilateral, la circunvolución temporal superior de forma bilateral y la circunvolución temporal media izquierda.

En un estudio realizado por Mazoyer et al. ${ }^{(10)}$, en donde los participantes escuchaban historias de forma pasiva, se mostró que ninguna de las regiones frontales derechas se activaron, a diferencia de lo que exponen Robertson et $\mathrm{al}^{\left({ }^{(11)}\right.}$ y St. George et al ${ }^{(12)}$. La ausencia de activación frontal derecha también fue observada por Tzourio et $\mathrm{al}^{(9)}$. Esto sugiere que la activación del hemisferio derecho podría aparecer sólo cuando el participante está armando una representación de la historia, en vez de procesar sonidos del lenguaje de forma pasiva.

Existe un estudio que determinó las diferencias de lateralización del lenguaje entre hombres y mujeres, mostrando que ambos sexos presentan activación bilateral de la corteza auditiva primaria y la circunvolución temporal superior, cuando el contraste en la estimulación se hace mediante una narración versus silencio ${ }^{(13)}$. Un segundo estudio que consistió en escuchar una narración durante periodos de activación y la misma narración reproducida al revés en periodos de reposo, arrojó activación bilateral de la circunvolución temporal media en las mujeres y activación izquierda de las circunvoluciones temporal media y superior en los hombres. Por último, se obtuvo activación de la circunvolución temporal superior izquierda y de la circunvolución temporal media derecha en ambos sexos, tras estimular mediante una narración versus la misma narración presentada de forma desordenada. Estos resultados permiten establecer una fuerte asimetría de las áreas posteriores del lenguaje en hombres y no en mujeres, lo que concuerda con trabajos como el de Hamposon y Kimura ${ }^{(14)}$.

El objetivo de este trabajo es implementar en un Hospital de Chile un protocolo de estimulación para conocer las áreas cerebrales involucradas en el procesamiento del lenguaje oral, en aspectos cognitivamente más complejos que los que involucra la comprensión de palabras o frase, utilizando estimulación auditiva. Se presentan aquí los resultados preliminares de este trabajo.

\section{Materiales y método}

Participaron 7 voluntarios sanos con audiometría normal, 3 hombres y 4 mujeres, con $23 \pm 1.7$ años de edad promedio, todos diestros. Para determinar la preferencia con que los voluntarios ejecutaban diferentes funciones, se les aplicó el test de lateralidad Edinburgh Handedness Inventory ${ }^{(15)}$.

El estudio contó con la aprobación de los Comités de ética locales y los voluntarios entregaron consentimiento informado por escrito.

Las adquisiciones fueron realizadas en el resona- dor magnético de la Unidad de Imaginología compleja del Hospital Van Buren, utilizando un equipo General Electric (GE) modelo Signa Excite LX, de 1,5 T, con la participación de los tecnólogos médicos de dicha institución.

Para generar la estimulación, se contó con un $P C$ equipado con Windows $X P$, equipo de música y un programa que permitió armar la secuencia de estimulación ${ }^{(16)}$. La historia utilizada para estimular auditivamente fue descargada desde un buscador de audio y corresponde a un extracto de un cuento infantil.

Para conducir el sonido desde la sala de control hacia el resonador se utilizó un transductor de señal eléctrica a neumática y un par de tubos de silicona, para evitar artefactos en la imagen generados por los cables eléctricos.

Debido a la pérdida de volumen generada por la longitud de los tubos, fue necesario amplificar la señal mediante un equipo de música. De esta forma, la etapa de estimulación estuvo compuesta por el PC desde donde se generó la estimulación, el transductor de señal eléctrica a neumática, el equipo de sonido, los tubos de silicona y los audífonos del resonador, por donde finalmente se presentó el estímulo a una intensidad promedio de $90 \mathrm{~dB}$ con una mínima de 77 $\mathrm{dB}$ y una máxima de $101 \mathrm{~dB}$, valores que corresponden a las mediciones realizadas con un equipo para medir intensidad de sonido.

Estimulación. El procedimiento aplicado a cada voluntario consistió en estimular por bloques mediante una historia reproducida en forma normal durante periodos de activación y la misma historia reproducida al revés durante períodos de reposo. Para poder distinguir de mejor forma el estímulo auditivo en el resonador, se filtró el audio de la historia realzando las voces de la narración y los personajes con un filtro pasabandas, mediante una de las opciones del programa de audio, y se minimizó el volumen de la música de fondo del cuento. En total, el protocolo estuvo formado por 4 períodos de estimulación y 5 períodos de reposo. La duración de ambos períodos fue de 40 segundos, a excepción del primer bloque de reposo el cual fue de 1 minuto con el fin de descontar los primeros 20 segundos de adquisición y así lograr una señal estable de T1, ya que a menos que el TR sea muy largo (aproximadamente 8 segundos) el contraste será ligeramente diferente en los primeros cortes de la adquisición antes de que la magnetización longitudinal haya alcanzado su estabilidad ${ }^{(17)}$.

La duración total de la prueba fue de 6 minutos 20 segundos. Previo a cada prueba, se les indicó a los voluntarios que escucharían una historia y que se les realizaría un cuestionario cuando ésta finalizara. Este cuestionario consistió en preguntas simples relacionadas con el contenido de lo que escucharon en los ciclos de activación, lo que permitió conocer lo que los voluntarios lograron entender de la tarea realizada, además de mejorar el rendimiento y la atención durante 
la prueba ${ }^{(18,19)}$. También se les solicitó que permanecieran inmóviles durante la adquisición, con los ojos cerrados, y que pusieran atención exclusivamente al estímulo presentado. Adquisición. Junto con la estimulación, se realizó una adquisición Echo Planar Imaging (EPI) de 8 cortes axiales, cubriendo la totalidad del lóbulo temporal con el fin de registrar el máximo de áreas involucradas con el procesamiento del lenguaje. Los parámetros utilizados fueron los siguientes: TR 4000 $\mathrm{ms}$, TE: $60 \mathrm{~ms}$, espesor de corte $7 \mathrm{~mm}$, espaciado 3 $\mathrm{mm}$, FOV $20 \times 20 \mathrm{~cm}^{2}$ y tamaño de matriz $64 \times 64$.

Una vez finalizadas las adquisiciones funcionales, se adquirió un volumen anatómico de alta resolución mediante una secuencia axial T 3D, con los siguientes parámetros: FOV $24 \mathrm{~cm}$, matriz de 512×192 pixeles, flip angle de $20^{\circ}$ y secuencia SPGR (Spoiled Gradient Echo).

Procesamiento de imágenes. Con el fin de conocer las activaciones registradas, las imágenes fueron procesadas temporal y espacialmente utilizando SPM5 (Statistical Parametrical Mapping) desarrollado por Wellcome Department of Imaging Neuroscience, University College London ${ }^{(20)}$.

El primer paso que se realizó fue la segmentación de las imágenes anatómicas en materia blanca y gris. Las imágenes funcionales fueron corregidas temporalmente, realineadas entre sí, co-registradas con las imágenes anatómicas y suavizadas mediante un kernel gaussiano de $6 \mathrm{~mm}$ (FWHM). Tanto las imágenes funcionales como anatómicas fueron normalizadas utilizando el estándar Montreal Neurological Institute (MNI). Una vez finalizadas las etapas anteriores, se realizó el análisis estadístico de las imágenes funcionales, el cual consistió en utilizar pruebas del tipo
Student t-test con p de 0.05, mediante la opción FWE (family wise error) de SPM5, lo que implica un 5\% de probabilidad de obtener falsos positivos. Como criterio de exclusión, se descartaron aquellas adquisiciones que presentaron activaciones no coherentes, es decir fuera de la corteza cerebral, $y / o$ aquellas que presentaron movimientos superiores a las dimensiones de un voxel.

En cada uno de los resultados de activación obtenidos se localizó el voxel de mayor activación (t-score) y se calculó la variación de señal registrada, que consiste en promediar los valores de intensidad obtenidos en las adquisiciones hechas en los periodos ON y los periodos OFF realizados en cada ciclo de estimulación, calculando la razón existente entre ellos de forma porcentual. Para el despliegue de las imágenes, se utilizó la opción slices de SPM5, que entrega 3 cortes axiales en formato radiológico.

\section{Resultados}

Todos los voluntarios entendieron la historia y las preguntas realizadas, respondiendo de forma correcta el cuestionario, tolerando satisfactoriamente el procedimiento en el escáner. En el $100 \%$ de los casos, los voluntarios fueron capaces de distinguir la historia presentada y respondieron de forma adecuada al cuestionario realizado al final de cada prueba.

La efectividad obtenida fue de un $71 \%$, es decir en cinco de siete pruebas realizadas fue posible registrar activaciones coherentes. La ubicación espacial del voxel de mayor activación presentó gran variación entre los voluntarios y los volúmenes obtenidos fueron pequeños y acotados a las áreas de interés, obteniendo un promedio de $5.06 \pm 2.99 \mathrm{~cm}^{3}$ (Tabla I).

Tabla I. En la tabla se muestran los resultados obtenidos para cada voluntario, organizados de acuerdo al hemisferio en donde se presentó el voxel de mayor activación. Además, en cada caso se presentan las activaciones y volúmenes en torno al voxel mayormente activado.

\begin{tabular}{|c|c|c|c|c|c|c|c|}
\hline \multirow[t]{2}{*}{$\begin{array}{l}\text { Volumen } \\
\text { y sexo }\end{array}$} & \multirow[t]{2}{*}{ t. score } & \multirow[t]{2}{*}{$\begin{array}{l}\text { Var. de } \\
\text { señal (\%) }\end{array}$} & \multicolumn{2}{|c|}{$\begin{array}{l}\text { Ubicación activación } \\
\text { en } \mathrm{mm} \text {. [x y z] }\end{array}$} & \multicolumn{2}{|c|}{$\begin{array}{l}\text { Volumen de activación } \\
\qquad\left(\mathrm{cm}^{3}\right)\end{array}$} & \multirow[t]{2}{*}{ Activaciór } \\
\hline & & & Hemis. Izq. & Hemis. Der. & Hemis. Izq. & Hemis. Der. & \\
\hline $1[\mathrm{~F}]$ & 6.08 & 0.48 & -- & $48-24-4$ & 2.25 & 4.71 & $\mathrm{CH}$ \\
\hline $2[\mathrm{M}]$ & 6.68 & 0.63 & -52648 & -- & 4.24 & -- & $\mathrm{CH}$ \\
\hline $3[\mathrm{~F}]$ & 6.60 & 0.49 & $-60-4432$ & -- & 4.24 & -- & W \\
\hline $4[\mathrm{M}]$ & 6.41 & 0.36 & $-50-364$ & -- & 10.93 & -- & W \\
\hline $5[\mathrm{~F}]$ & 6.04 & 0.36 & -- & $62-6-8$ & -- & 4.03 & CTM \\
\hline $\begin{array}{l}\text { Promedio } \\
{[\mathrm{x}]} \\
{[\mathrm{y}]} \\
{[\mathrm{z}]}\end{array}$ & $6.3 \pm 0.3$ & $0.47 \pm 0.11$ & $\begin{array}{l}-54 \pm 5.3 \\
-24 \pm 27 \\
28 \pm 22\end{array}$ & $\begin{array}{l}55 \pm 9.8 \\
-15 \pm 2.7 \\
-6 \pm 2.8\end{array}$ & $5.4 \pm 3.8$ & $4.3 \pm 0.5$ & \\
\hline
\end{tabular}




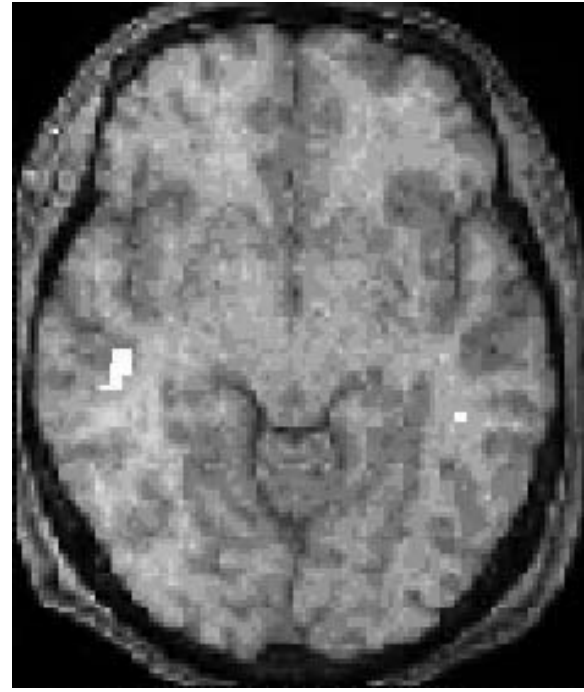

Figura 1. Activación circunvolución de Heschl derecha.

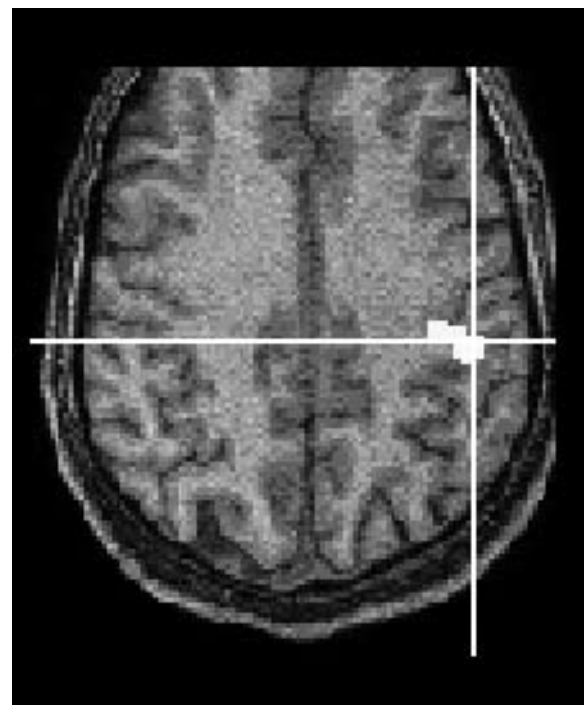

Figura 2. Activación circunvolución de Heschl izquierda.

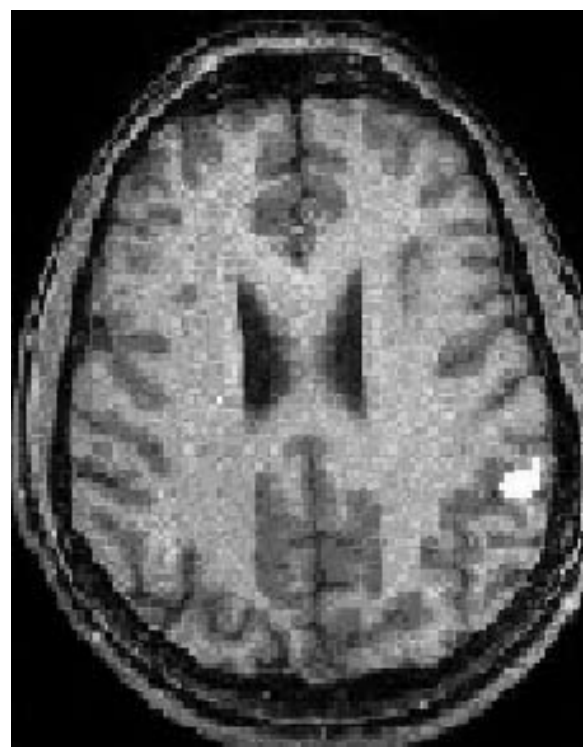

Figura 3. Activación área de Wernicke.

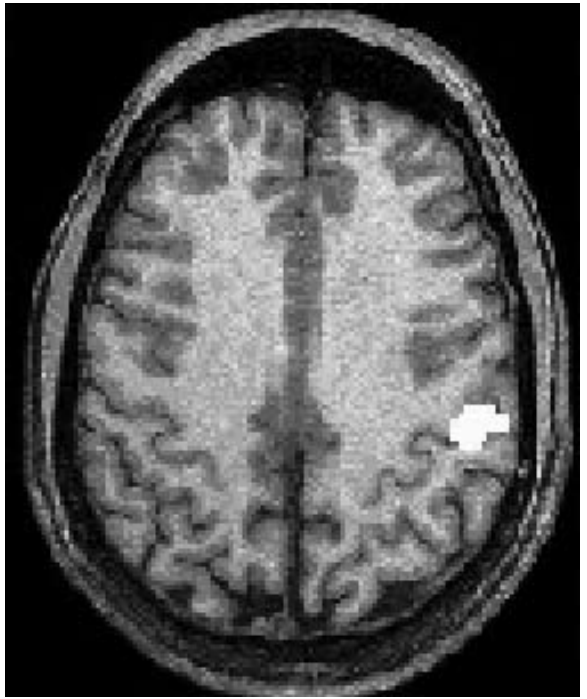

Figura 4. Activación área de Wernicke.

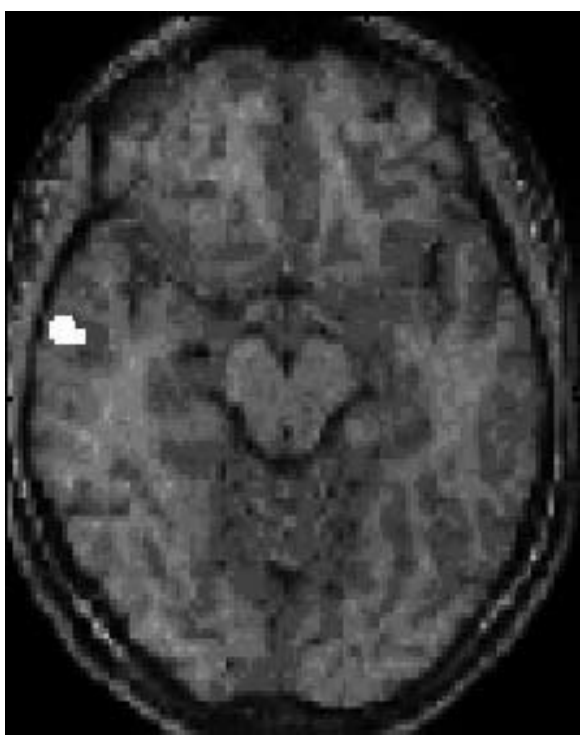

Figura 5. Activación circunvolución temporal media derecha.

La variación de señal fue baja, registrando un valor de $0.5 \pm 0.1 \%$, hecho que puede deberse a la baja relación señal ruido de las adquisiciones, a la dificultad cognitiva de la prueba y al ruido acústico constante de la adquisición, que ha demostrado ser un factor importante en el enmascaramiento de las activaciones generadas por la verdadera estimulación $^{(21)}$. Las áreas corticales obtenidas mediante el protocolo seleccionado dieron cuenta de diferentes regiones asociadas a la comprensión del lenguaje. Además del área de Wernicke, las activaciones se presentaron en la circunvolución temporal media y circunvolución de Heschl, de forma bilateral (Figuras 1-5). 


\section{Discusión}

El protocolo para localizar las áreas corticales involucradas en la comprensión del lenguaje auditivo fue validado en cinco de siete voluntarios sanos de manera satisfactoria, es decir, una efectividad de un $71 \%$.

El t-score alcanzado fue de $6.3 \pm 0.3$, un valor relativamente bajo en relación a trabajos implementados anteriormente en el mismo Hospital, como los casos de RMf motor y auditivo primario en donde se obtuvo valores promedio de 14,25 y 11,59 respectivamente ${ }^{(22,23)}$. Sin embargo, se debe tener en cuenta que el área motora, al igual que las regiones auditiva y visual, representan regiones mucho más definidas y localizadas, que cuales entregan mejor variación de señal y nivel de activación que las áreas del lenguaje, las cuales son más extensas, menos acotadas y en esta zona las tareas de estimulación poseen una complejidad cognitiva menor a las de comprensión del lenguaje. Al respecto, si se comparan estos resultados con los de un trabajo anterior sobre detección de corteza auditiva primaria ${ }^{(23)}$ que posee características de adquisición similares a las utilizadas en este trabajo, se puede verificar que la estimulación con palabras generó un mayor volumen de activación, variación de señal y significancia estadística que lo obtenido en la comprensión de la historia $\left(58.93 \pm 35.61 \mathrm{~cm}^{3}, 1.19 \pm 0.26\right.$ y $11.59 \pm 3.68$, respectivamente).

EI SNR de las adquisiciones fue de 23:1 en cerebro; se midió calculando el promedio de una región de materia blanca en las imágenes anatómicas (señal) y dividiendo este valor por la desviación estándar de otra región en la imagen que no contiene información (ruido). Este resultado representa un valor bajo, del orden de la mitad de lo que sería una imagen con buena tasa de señal a ruido ${ }^{(24)}$. En RMf es de gran importancia poder contar con valores de SNR altos, con el fin de poder detectar los cambios de intensidad de la imagen como resultado de la estimulación. En este sentido, si el valor de SNR es bajo, la presencia de ruido en las imágenes será mayor, dificultando la detección de activaciones en los voxeles en el tiempo. A pesar de la dificultad de trabajar con un resonador cuyas imágenes presentan baja tasa de señal a ruido, se ha logrado obtener resultados interesantes de activación funcional para una tarea de comprensión de lenguaje oral.

Los resultados obtenidos en este estudio coinciden con los obtenidos en otros trabajos que han estudiado la comprensión del lenguaje auditivo. En un estudio realizado en el 2000, Kenji Kansaku ${ }^{(13)}$, investigó las diferencias de activación en el procesamiento del lenguaje entre hombres y mujeres, utilizando el mismo contraste de estimulación que el usado en este estudio. Los resultados obtenidos coinciden con los de este trabajo, ya que se logró obtener en ambos sexos activación de la circunvolución temporal media y área de Wernicke. Si bien el volumen de activación promedio obtenido en el estudio conducido por Kenji Kansaku fue cinco veces mayor al de este estudio, se debe tomar en cuenta que se utilizó un equipo de $3 \mathrm{~T}$, lo que permite tener una mejor relación señal a ruido.

Un hecho importante al momento de idear el tipo de prueba realizado en este estudio, es considerar una distribución lo más similar posible del volumen y frecuencias entre los períodos de activación y reposo, con el fin de evitar que las activaciones se deban a estímulos auditivos. Al respecto, se tuvo especial cuidado en homogenizar las frecuencias del estímulo tanto en los períodos de activación como de reposo. Además, el contraste de estimulación empleado permitió que las activaciones fueran netamente debidas a aspectos de comprensión de lenguaje y no auditivas.

\section{Conclusión}

Los resultados obtenidos en este estudio fueron satisfactorios, a pesar de la baja relación señal a ruido de las imágenes y la dificultad que implica en general realizar estudios mediante resonancia funcional auditiva, donde existen limitaciones importantes relacionadas principalmente con el ruido acústico constante de las adquisiciones. Además, se debe destacar que el estudio se realizó utilizando únicamente las herramientas disponibles en la Unidad de Imagenología del Hospital, sin la necesidad de adquirir otros implementos.

Las activaciones obtenidas constituyen un primer avance en el estudio de las áreas involucradas en la comprensión del lenguaje mediante RMf. Al respecto, cabe mencionar la importancia de realizar un número mayor de pruebas, que permitan identificar las áreas involucradas en este tipo de estimulación y utilizar los resultados de RMf de manera confiable en aplicación clínica, como por ejemplo en pacientes aquejados de tumores, con el fin de conocer la real utilidad del protocolo para la planificación neuroquirúrgica.

\section{Agradecimientos}

Los autores agradecen el apoyo del proyecto FONDECYT 11060036 y también quieren agradecer la colaboración de los tecnólogos médicos: Juan Almuna, Hugo Cahuana, Cristian Morales y Hugo Moroso.

\section{Bibliografía}

1. Kwong KK, Belliveau JW, Chesler DA, Goldberg IE, Weisskoff RM, Poncelet $P$ et al. Dynamic magnetic resonance imaging of human brain activity during primary sensory stimulation. Proceedings of the National Academy of Sciences (USA) 1992; 89: 5675-9.

2. Jezzard et. al, Jezzard P, Matthews P, Smith S. "Functional MRI: An Introduction to Methods", 1st ed, 
New York, Oxford University Press2001.

3. Petrella. "Preoperative functional MR imaging localization of language and motor areas: effect on therapeutic decision making in patients with potentially resectable brain tumors". Radiology 2006; 240: 793-802.

4. Chee MW, O'Craven KM, Bergida R, Rosen BR, Savoy $\mathrm{RL}$. Auditory and visual word processing studied with fMRI. Hum. Brain Mapp 1999; 7: 15-28.

5. Pugh KR, Shaywitz BA, Shaywitz SE, Constable RT, Skudlarski $P$, Fulbright $R$ et al. Cerebral organization of component processes in reading. Brain 1996; 119: 1221-38.

6. Petersen SE, Fox PT, Posner MI, Mintun M, Raichle ME. 1989. Positron emission tomographic studies of the processing of single words. J Cogn Neurosci 1989; 1: 153-70.

7. Desmond JE, Sum JM, Wagner AD, Demb JB, Shear $\mathrm{PK}$, et al. Functional MRI measurement of language lateralization in Wadatested patients. Brain 1995; 118: 155-78.

8. Kiehl KA, Liddle PF, Smith AM, Mendrek A, Forster BB, Hare RD. Neural pathways involved in the processing of concrete and abstract words. Hum. Brain Mapp1999; 7: 225-33.

9. Tzourio N, Nkanga-Ngila B, Mazoyer B. Left planum temporale surface correlates with functional dominance during story listening. NeuroReport 1998; 9: 829-33.

10. Mazoyer BM,Tzourio N, FrakV, Syrota A, Murayama N, et al. The cortical representation of speech. J Cogn Neurosci 1993; 5: 467-79.

11. Robertson DA. 2000. Functional neuroanatomy of language comprehension. PhD diss., Univ. Wis., Madison

12. St. George M, Kutas M, Martinez A, Sereno MI. Semantic integration in reading: engagement of the right hemisphere during discourse processing. Brain 1999; 122: 1317-25.

13. Kansaku K, Yamaura A, Kitazagua S. Sex differences in lateralization revealed in the posterior language areas. Cerebral cortex Sep 2000; 10: 866-872.

14. Hampson E, Kimura D. Sex differences and hormonal influences on cognitive functions in humans. Behavorial endocrinology1992; Vol: 357-358, Cambridge, Ma, MIT press.

15. Disponible en hyyp;//www.neuropsicol.org/Protocol/ Oldfield.pdf

16. Audacity 1,2.6._Disponible en http://audacity.sourceforge.net/

17. Jezzard $P$, Matthews $P$, Smith $S$. "Functional MRI: An Introduction to Methods", 1st ed, New York, Oxford University Press, 2001.

18. Binder JR, Frost J, Hammeke TA, Rao SM, Cox RW. "Function of the left planum temporale in auditory and linguistic processing". Brain 1996; 119: 1239-1247.

19. Chen J, Penhuene V, Zatorre R. "Tapping in Synchrony to Auditory Rhythms". Annals of the New York Academy of Sciences 2005; 1060: 400-403. New York Academy of Sciences.

20. SPM5. Disponible en http://www.fil.ion.ucl.ac.uk./spm/ software/spm5/

21. Talavage TM, Edmister WB, Ledden PJ, Weisskoff RM. "Comparison of impact of fMRI sequence acoustics on auditory cortex activation". In: Proceedings of the 6th Annual meeting of the International Society for Magnetic Resonance in Medicine.p 1503.

22. Marco Morán Araus, 2008. "Implementación de Imagenología Funcional por Resonancia Magnética para Pruebas Motoras en el Hospital Carlos Van Buren". Tesis de Pregrado Ingeniería Biomédica.

23. Ignacio Letelier Aguirre, 2009. "Implementación y validación de protocolos de estimulación auditiva primaria y de comprensión del lenguaje oral por resonancia magnética funcional en el Hospital Carlos Van Buren". Tesis de Pregrado Ingeniería Civil Biomédica.

24. Haacke M, Brown R, Thompson M, Venkatesan R. Magnetic Resonance Imaging: Principles and Sequence Design, Wiley-Liss, 2000. 